\title{
On the Optimal Convergence
}

\author{
M. STOJANOVIĆ
}

Two classes of second order optimally convergent 3 -point difference schemes for singularly perturbed self-adjoint problem are presented. They achieve $O\left(\min \left(h^{2}, \varepsilon\right)\right)$ point-wise error estimates and $O\left(h^{2}\right)$ global ones.

hey words: Optimal convergence, singularly perturbed problems

AMS subject classification : $65 \mathrm{~L} 10$

\section{Introduction}

Hemker gives in [3] a brief survey and the main directions and references of the main problems that are encountered when singular perturbation problems are solved by numerical means in one dimension, and opens some areas of current research in more dimensions. He lists the known standard techniques to obtain numerical approximation which describe the solution sufficiently accurate. Much efforts can be spent on the construction and analysis of $\varepsilon$-uniform difference schemes, where $\varepsilon$ is a small parameter, $0<\varepsilon \leq 1$, and some results have been obtained by many authors [1, 3, 4]. Uniform convergence was a strong criterion of convergence for difference schemes.

We recall the definition of this criterion.

Denote by $u_{i, \varepsilon, h}$ the computed solution of a singularly perturbed problem and by $u_{\varepsilon}\left(x_{i}\right)$ the exact one at points $x_{i}, i=0(1) n$, where $h$ is the step size of discretization which computes the approximate solution and $\varepsilon$ is a small parameter, $0<\varepsilon \leq 1$. Suppose that the mesh $\Delta$ of discretization is uniform: $0=x_{0}<x_{1}<\ldots<x_{n}=1, h=1 / N, N$ being an integer, $x_{i}=x_{i-1}+h, i=$ $1(1) n$. Then we can give the following

Definition 1 (see [1]): The error bound of the form

$$
\left|u_{i, \varepsilon, h}-u_{c}\left(x_{j}\right)\right| \leq M h^{9}
$$

holds uniformly in $\varepsilon$ of order $q$ if the constant $M$ does not depend on both $h$ and $\varepsilon$.

The concept of optimality was introduced in [1] to impose a stricter criterion.

Definition 2 (see [2]): The error bound of the form

$$
\left|u_{i, \varepsilon, h}-u_{\varepsilon}\left(x_{i}\right)\right| \leq M \min \left(\max _{0 \leq j \leq i}\left(h_{j}^{\rho}, \varepsilon\right)\right)
$$

holds optimaly in $s$ of order $\rho$ if the constant $M$ does not depend on both $h$ and $s$. 
In [2] Farrel examined sufficient conditions for uniform and optimal convergence for a class of difference schemes for stiff initial value problems. He showed that there exists a subclass of schemes which is optimal in the sense that

$\left|u_{i, \varepsilon, h}-u_{\varepsilon}\left(x_{j}\right)\right| \leq M \min (h, \varepsilon), M$ independent on both $h$ and $\varepsilon$.

Also, he showed that higher order optimal schemes exist. In [5] an optimal difference scheme of order one is given for a self-adjoint equation, by splines in tension.

Here we develop the theory of optimality for difference schemes for a singularly perturbed self-adjoint problem. In the text to follow, $M$ will denote different constants independent of both parameters $h$ and $\varepsilon$, and $\tau_{j}$ will denote the truncation error of discretization. Indices $h$ and $\varepsilon$ in $u$ will be omitted.

The paper is organized as follows. In the first part we give the construction of the two classes of difference schemes which have optimal second order error estimates for the problem

$$
\begin{aligned}
& L u:=-\varepsilon u^{\prime \prime}+\rho(x) u=f(x) \\
& u(0)=A, u(1)=B, 0<\varepsilon \leq 1,
\end{aligned}
$$

where $p$ and $f$ are continuously differentiable at least of two. We treat it numerically by solving a system of equations. In the second part we provide an error bound in maximum norm and present an outline of the proofs for the optimal convergence at nodes, and for the global one in the third part. In the fourth part we test both classes of schemes on numerical examples to illustrate the formulae developed.

\section{Construction of the schemes}

This part of the paper is concerned with the following two subjects:

1. The construction of second order optimally convergent schemes using the solution of the problem

$$
-\varepsilon S_{\triangle_{i}}^{\prime \prime}+\bar{p}_{i} S_{\triangle_{j}}=\bar{f}_{i}, S_{\triangle_{j}}\left(x_{i}\right)=u_{i}, S_{\triangle_{i}}\left(x_{i-1}\right)=u_{j-1}
$$

on each subinterval $\Delta_{i}=\left[x_{i}, x_{i}\right], i=1(1) n$, of the interval $[0,1]$, where $\bar{p}_{i}, \bar{f}_{i}$ are piecewise constants.

2. Generation of schemes using the solution of the problem

$$
-\varepsilon S_{\triangle_{i}}^{\prime \prime}+\bar{p}_{i} S_{\triangle_{i}}=\frac{\left(x-x_{i-1}\right)}{h} f_{j}+\frac{\left(x_{i}-x\right)}{h} f_{i-1}, S_{\triangle_{i}}\left(x_{i}\right)=u_{i}, S_{\triangle_{j}}\left(x_{i-1}\right)=u_{i-1},
$$

where $\bar{p}_{i}$ is piecewise constant and $f_{i}=f\left(x_{i}\right), u_{i}, u_{i-1}$ are the approximate solution for (1) which will be determined from the corresponding difference schemes.

In both cases our assumptions on $S_{\triangle_{i}}$ will be the following ones:

a) The first derivative of the splines $S_{\triangle_{i}}$ is continuous at mid points, i.e., $S_{\triangle_{i}}\left(x_{i}+h / 2\right)=$ $S_{\Delta_{i+1}}\left(x_{i}+h / 2\right)$.

b) The spline has nodes at mesh points.

i) The first class of schemes. Solving (2) we obtain 


$$
\begin{aligned}
& S_{\triangle_{i}}(x)=\bar{f}_{i} / \bar{p}_{i}+\frac{\exp \left(\sqrt{\bar{p}_{i} / \varepsilon}\left(x-x_{i}\right)\right)}{2 \operatorname{sh} \rho_{i}}\left\{\exp p_{i}\left(u_{i}-\bar{f}_{i} / \bar{p}_{i}\right)-\left(u_{i-1}-\bar{f}_{i} / \bar{p}_{i}\right)\right\} \\
& +\frac{\exp \left(-\sqrt{\bar{p}_{i} / \varepsilon}\left(x-x_{i-1}\right)\right)}{2 \operatorname{sh} \rho_{i}}\left\{\exp p_{i}\left(u_{i-1}-\bar{f}_{i} / \bar{p}_{i}\right)-\left(u_{i}-\bar{f}_{i} / \bar{p}_{i}\right)\right\} \text {. }
\end{aligned}
$$

From the smoothness of the first derivative at the point $x=x_{i}+h / 2$ we obtain the class of difference schemes

$$
R u_{i}=Q \bar{f}_{j}, u(0)=A, u(1)=B
$$

referred to the approximation to the functions $f$ and $\rho$, which can be set. In (5) we have

$$
\begin{aligned}
& R u_{i}=r_{i} u_{i-1}+r_{i}^{c} u_{i}+r_{i}^{+} u_{j+1}, Q \bar{f}_{i}=\bar{q}_{i}^{-} \bar{f}_{i}+\bar{q}_{i}^{+} \bar{f}_{i+1} \\
& r(\rho)=\rho(\operatorname{sh} \rho)^{-1} \\
& r_{j}^{-}=r\left(\rho_{i}\right) \operatorname{ch}\left(\rho_{i} / 2\right), r_{i}^{c}=-r\left(\rho_{i}\right) \operatorname{ch}\left(3 \rho_{j} / 2\right)-r\left(\rho_{i+1}\right) \operatorname{ch}\left(\rho_{i+1} / 2\right), r_{i}^{+}=r\left(\rho_{i+1}\right) \operatorname{ch}\left(\rho_{i+1} / 2\right) \\
& \bar{q}_{i}^{-}=-1 / \bar{\rho}_{i} 2 \rho_{i} \operatorname{sh}\left(\rho_{i} / 2\right), \bar{q}_{i}^{+}=0, \rho_{i}=\sqrt{\bar{\rho}_{i} / \varepsilon} h, \rho_{i}=\rho\left(x_{i}\right) .
\end{aligned}
$$

For $p$ (analogously for $f$ ) we use a piecewise constant approximation of the form

$$
p=\sum_{i=1}^{n} \bar{p}_{i}\left(x_{i}-x\right)_{+}, \text {where }(\cdot)_{+} \text {is the cutoff symbol: } x_{+}=\left\{\begin{array}{l}
x \text { for } x>0 \\
0 \text { for } x \leq 0
\end{array} .\right.
$$

Consider two different approximations for $p$ and $f$ which imply two different schemes:

1. The approximation $2\left({ }^{-}\right)_{j}=(\cdot)_{j-1 / 2}+(\cdot)_{j+1 / 2}$ for $p$ and $f$, respectively, gives the scheme

$$
R u_{i}=Q f_{i}
$$

where $Q f_{i}=q_{j}^{-} f_{i-1 / 2}+q_{i}^{+} f_{i+1 / 2}, q_{j}^{-}=1 / 2 \bar{q}_{i}^{-}, q_{i}^{+}=1 / 2 \bar{q}_{i}^{-}$and $R u_{i}$ is given in (5).

2. The approximation 6()$\left.^{-}\right)_{j}=(\cdot)_{i-1 / 2}+4(\cdot)_{i}+(\cdot)_{i+1 / 2}$ for $p$ and $f$, respectively, gives the scheme

$$
R u_{j}=Q f_{j}
$$

ii) The second class of schemes. The solution of (3) is a well-known tension spline [5]. Applied to (2) it yields

$$
\begin{aligned}
S_{\triangle_{i}}(t)= & u_{i}\left\{t+\frac{\bar{p}_{i}}{\varepsilon} \frac{h^{2}}{\rho_{i}^{2}}\left(\frac{\operatorname{sh}\left(\rho_{i} t\right)}{\operatorname{sh} \rho_{i}}-t\right)\right\} \\
& +u_{i-1}\left\{(1-t)+\frac{\bar{\rho}_{i}}{\varepsilon} \frac{h^{2}}{\rho_{i}^{2}}\left(\frac{\operatorname{sh}\left(\rho_{i}(1-t)\right)}{\operatorname{sh} \rho_{j}}-(1-t)\right)\right\} \\
& -\frac{\bar{f}_{i}}{\varepsilon} \frac{h^{2}}{\rho_{i}{ }^{2}}\left(\frac{\operatorname{sh}\left(\rho_{i} t\right)}{\operatorname{sh} \rho_{i}}+\frac{\operatorname{sh}\left(\rho_{i}(1-t)\right)}{\operatorname{sh} \rho_{j}}-1\right), i=1(1) n .
\end{aligned}
$$

The continuity condition of the first derivative at the mid points gives the class of difference schemes

$$
R u_{j}=Q \bar{f}_{i}
$$


where

$$
\begin{aligned}
& r_{i}^{-}=1+\left(r\left(\rho_{i}\right) \operatorname{ch}\left(\rho_{i} / 2\right)-1\right), r_{i}^{+}=1+\left(r\left(\rho_{i+1}\right) \operatorname{ch}\left(\rho_{i+1} / 2\right)-1\right) \\
& r_{i}^{c}=-2-\left(r\left(\rho_{i}\right) \operatorname{ch}\left(3 \rho_{i} / 2\right)-1\right)-\left(r\left(\rho_{i+1}\right) \operatorname{ch}\left(\rho_{i+1} / 2\right)-1\right) \\
& \bar{q}_{i}^{-}=1 / \bar{\rho}_{i-1}\left(r\left(\rho_{i}\right) \operatorname{ch}\left(\rho_{i} / 2\right)-r\left(\rho_{i}\right) \operatorname{ch}\left(3 \rho_{j} / 2\right)\right) \\
& \bar{q}_{i}^{+}=1 / \bar{\rho}_{i+1}\left(r\left(\rho_{i+1}\right) \operatorname{ch}\left(\rho_{i+1} / 2\right)-r\left(\rho_{i}\right) \operatorname{ch}\left(\rho_{i} / 2\right)\right) \\
& \rho_{i}=\sqrt{\bar{\rho}_{i} / \varepsilon} h, r(\rho)=\rho(\operatorname{sh})^{-1} .
\end{aligned}
$$

Here, the schemes referred also to the approximation to the functions $f$ and $p$.

The schemes which uses the approximation

$$
\text { 1. } \left.2\left({ }^{-}\right)_{i}=(\cdot)_{j-1 / 2}+(\cdot)_{j+1 / 2} \text { and } 2.6()^{-}\right)_{j}=(\cdot)_{i-1 / 2}+4(\cdot)_{i}+(\cdot)_{i+1 / 2} \text {. }
$$

for driving terms in (1) are listed below, respectively:

$$
\begin{aligned}
& Q f_{i}=q_{i-1 / 2}^{-} f_{j-1 / 2}+q_{i+1 / 2}^{+} f_{i+1 / 2}+q_{i+3 / 2}^{+} f_{i+3 / 2} \\
& q_{i 1 / 2}^{-}=1 /\left(2 \bar{p}_{i}\right)\left(r\left(\rho_{i}\right) \operatorname{ch}\left(\rho_{i} / 2\right)-r\left(\rho_{j}\right) \operatorname{ch}\left(3 \rho_{i} / 2\right)\right) \\
& q_{i+3 / 2}^{+}=1 /\left(2 \bar{p}_{i+1}\right)\left(r\left(\rho_{i+1}\right) \operatorname{ch}\left(\rho_{i+1} / 2\right)-r\left(\rho_{j}\right) \operatorname{ch}\left(\rho_{j} / 2\right)\right) \\
& q_{i+1 / 2}^{+}=q_{i-1 / 2}+q_{i+3 / 2}^{+} \\
& q_{i-1 / 2}^{+}=1 /\left(6 \bar{p}_{i}\right)\left(r\left(\rho_{i}\right) \operatorname{ch}\left(\rho_{j} / 2\right)-r\left(\rho_{j}\right) \operatorname{ch}\left(3 \rho_{j} / 2\right)\right) \\
& q_{j}^{\Gamma}=4 q_{i}^{-}{ }^{-1 / 2} \\
& q_{i+1 / 2}^{+}=1 /\left(6 \bar{p}_{i+1}\right)\left(r\left(\rho_{i+1}\right) \operatorname{ch}\left(\rho_{i+1} / 2\right)-r\left(\rho_{j}\right) \operatorname{ch}\left(\rho_{i} / 2\right)\right)+q_{j-1 / 2} \\
& q_{j+1}^{+}=4 q_{j+1 / 2}^{+}, \quad q_{i+3 / 2}^{+}=q_{i+1 / 2}^{+} .
\end{aligned}
$$

The left-hand side of equality $R u_{i}=Q f_{i}$ is the same as in (5).

\section{Optimal nodal error estimate}

Following the concept of optimality given in Definition 2, we obtain that for the schemes (5), (7) a stronger criterion of convergence holds. This is shown in the next three theorems.

Theorem 1: Let $p, f \in C^{2}[0,1]$ and $\left\{u_{i}\right\}_{i=1}^{n-1}$ be the solution of the discretization (5). Then the estimate

$$
\left|u_{i}-u\left(x_{i}\right)\right| \leq M \min \left(h^{2}, \varepsilon\right), i=0(1) n
$$

holds. Namely, the schemes (5) are optimal and of order 2.

However, to prove Theorem 1 we must define the argument in the following

Lemma 1 (see [1]): Let $p, f \in C^{2}[0,1]$ and $p^{\prime}(0)=p^{\prime}(1)=0$. Then the solution of (1) can be decomposed in three parts:

$$
u(x)=q_{0} u_{0}(x)+q_{1} u_{0}(x)+g(x),
$$


where $\left|q_{0}\right|,\left|q_{1}\right|$ are constants:

$$
\begin{aligned}
& \left|q_{0}\right|,\left|q_{1}\right| \leq M, u_{0}(x)=\exp \{-x \sqrt{p(0) / \varepsilon}\}, \omega_{0}(x)=\exp \{-(1-x) \sqrt{p(1) / \varepsilon}\}, x \in(0,1), \\
& \left|g^{(i)}(x)\right| \leq M\left(1+\varepsilon^{1-i / 2}\right), i=0(1) 4 .
\end{aligned}
$$

To accomplish the error estimate we consider $u_{0}, \omega_{0}$ and $g$ separately.

Lemma 2 (see [6]): Let $u \in C^{3}[0,1]$ be the exact sulution of (1). Then the truncation error of discretization $(5)_{1}\left(\tau_{i}(u)=R u_{j}-Q\left(L u_{i}\right)\right)$, for each of the functions from Lemma 1 separately, is the following:

1. $\tau_{i}(g)=T_{i}^{(0)} g+T_{i}^{(1)} g^{*}+T_{i}^{(2)} g^{\prime \prime}+T_{i}^{(3)} g^{\cdots}+\ldots+R$,

where

$$
\begin{aligned}
& T_{i}^{(0)}=r_{i}^{-}+r_{i}^{c}+r_{i}^{+}-\bar{p}_{i} q_{j}^{-}-\bar{p}_{i+1} q_{j}^{+} \\
& T_{i}^{(1)}=h\left(\left(r_{i}^{+}-r_{i}\right)+\bar{p}_{i} / 2 q_{i}^{-}-\bar{p}_{i+1} / 2 q_{i}^{+}\right) \\
& T_{i}^{(2)}=h^{2}\left(r_{i}^{+} / 2+r_{i}^{-} / 2-\bar{p}_{i} / 8 q_{i}^{-}-\bar{p}_{i+1} / 8 q_{i}^{+}\right)+\varepsilon\left(q_{i}^{+}+q_{i}^{-}\right) \\
& T_{i}^{(3)}=h^{3}\left(r_{j}^{+} / 6-r_{i}^{-} / 6+\bar{p}_{i} / 48 q_{i}^{-}-\bar{p}_{i+1} / 48 q_{i}^{+}\right)+\varepsilon h / 2\left(q_{i}^{+}-q_{j}^{-}\right) . \\
& \text {2. } \tau_{i}\left(u_{0}\right)=u_{0}\left(r_{i}^{-} \exp \left(\rho_{0}\right)+r_{i}^{c}+r_{i}^{+} \exp \left(-\rho_{0}\right)\right. \\
& \left.\quad-\left(p_{0}-p_{i-1 / 2}\right) \exp \left(p_{0} / 2\right) q_{i}^{-}-\left(p_{0}-p_{i+1 / 2}\right) \exp \left(-\rho_{0} / 2\right) q_{i}^{+}\right),
\end{aligned}
$$

where

$$
u_{o i}=\exp \left(-\sqrt{p(0) / \varepsilon} x_{i}\right), \rho_{0}=\sqrt{p(0) / s} h,
$$

and $r_{j}^{ \pm}: r_{i}^{c}, q_{i}^{ \pm}, j=1(1) n-1$ are the coefficients from $(5)_{1}$.

3. Similarly as in assertion 2 we obtain $\tau_{i}\left(\omega_{0}\right)$.

Lemma 3: Let $u \in C^{3}[0,1]$ be the exact sulution of $(1)$ and let $p, f \in C^{2}[0,1], p^{\prime}(0)=p \cdot(1)=$ 0 . Then we have the following consistency error of the discretization $(5)_{1}$ :

$$
\max _{i}\left\{\left|\tau_{i}(g)\right|,\left|\tau_{i}\left(u_{0}\right)\right|,\left|\tau_{i}\left(\omega_{0}\right)\right|\right\} \leq M\left\{\begin{array}{ll}
h^{1 / \varepsilon} & \text { if } h^{2} / \varepsilon<1 \\
\varepsilon p_{i} \exp p_{i} & \text { if } h^{2 / \varepsilon} \geq 1
\end{array}, i=1(1) n-1 .\right.
$$

Proof: Estimate for $\left|\tau_{j}(g)\right|$. In Lemina $2 / 1$ we have $T_{i}^{(0)}=T_{j}^{(1)}=0$, and $\left|T_{j}^{(2)} g \cdot\right|$ and remainders are less or equal to $M h^{4} / \varepsilon$ when $h^{2} / \varepsilon \leq 1$. In the opposite case, $h^{2} / \varepsilon \geq 1$, the largest term is $\varepsilon p_{i} \operatorname{sh}\left(p_{i} / 2\right)$.

Estimate for $\left|\tau_{j}\left(u_{0}\right)\right|$. Since $|p(x)-p(0)| \leq M x^{2}$ under the condition of Lemma 1 and $\left|q_{i}^{ \pm}\right|$s $M \rho_{i} \operatorname{sh} \rho_{i} / 2$ we obtain $\left|\tau_{i}\left(u_{o}\right)\right| \leq M \varepsilon \rho_{j} \exp \rho_{i}$ when $h^{2} / \varepsilon 21$. We obtain the estimate for $h^{2} / \varepsilon \leq 1$ after Taylor developments.

Estimate for $\left|\tau_{i}\left(\omega_{0}\right)\right|$. It is the same as the estimate for $\tau_{i}\left(u_{0}\right)$

Theorem 2: Denote by $\left\{u_{i}\right\}_{i=1}^{n-1}$ the approximate solution to the one $u \in C^{3}[0,1]$ of (1) obtained by the discretization $(\mathrm{S})_{1}$. If $p^{\circ}(0)=p^{\cdot(1)}$ holds and $p, f \in C^{2}[0,1]$, then

$$
\left|u_{i}-u\left(x_{i}\right)\right| \leq M / \min \left(h^{2}, \varepsilon\right), \quad i=o(1) n,
$$

i.e., the scheme (5), has the second order of optimal convergence.

9 Analysis. Bd. 11, Heft 1 (1992) 
Proof: Denote by $A \in \mathbb{R}^{n-1, n-1}$ the matrix of the system of equations (5). Then

$$
\|A\|^{-1} \leq\left(r_{i}^{-}+r_{j}^{\mathrm{c}}+r_{i}^{+}\right)^{-1} \leq M\left\{\begin{array}{ll}
\varepsilon / h^{2} & \text { if } h^{2} / \varepsilon \leq 1 \\
1 /\left(\rho_{j} \operatorname{sh}\left(\rho_{j} / 2\right)\right) & \text { if } h^{2 / \varepsilon} \geq 1
\end{array} .\right.
$$

The nodal errors are

$$
\max _{i}\left|u_{i}-u\left(x_{i}\right)\right| \leq\|A\|^{-1} \max _{i}\left|\tau_{i}(u)\right| .
$$

From Lemma 1 and Lemma 3 we obtain the estimate of the truncation error $\tau_{j}(u)=\tau_{j}\left(u_{0}\right)$ $+\tau_{j}\left(\omega_{0}\right)+\tau_{j}(g)$. Hence, $(8)$ and $(9)$ give the assertion of the theorem

Also, we obtain in the same way that the assertion of Theorem 1 holds in the case $(5)_{2}$, which completes the proof of Theorem 1. Similarly we have

Theorem 3: Let $p, f \in C^{2}[0,1]$ and $\left\{u_{i}\right\}_{i=1}^{n-1}$ be the approximate solution of (1) obtained by (7). If $p \cdot(0)=p^{\prime}(1)=0$, then

$$
\left|u_{i}-u\left(x_{i}\right)\right| \leq M \min \left(h^{2}, \varepsilon\right), j=0(1) n .
$$

On the other hand, the schemes (7) are optimal and of order two in the sense of Definition 2.

\section{Uniform global error estimate}

Firstly, we give the following

Theorem 4: Let $\left\{S_{\triangle_{i}}(x)\right\}$ be the spline solution (4) of (1) on each subinterval $\left[x_{i-1}, x_{i}\right]$. $i=1(1) n$, where the coefficients $\left\{u_{i}\right\}_{i=1}^{n-1}$ we obtain from the linear system (S). Let $p, f \in C^{2}[0,1]$ and $p \cdot(0)=p \cdot(1)=0$. Then the estimate

$$
\left|u(x)-S_{\triangle_{i}}(x)\right| \leq M h^{2}, i=1(1) n
$$

holds. It means that the schemes (5) have order two of uniform global error estimate.

Proof: Denote by $E S_{\triangle_{i}}$ the exponential part of the spline (4) and by $L S_{\triangle_{i}}$ the linear one. When $p=$ const then $E S_{\triangle_{i}}$ is in a kernel of a differential operator. Then $L\left(E S_{\Delta_{i}}(x)\right)=0$ and the global error depends on the approximation to the function $f$. Since $\left|f-\bar{f}_{i}\right| \leq M h^{2}$ then $\left|L\left(u-S_{\triangle_{i}}(x)\right)\right| \leq M h^{2}$. When $p \neq 0$ we have

$$
\begin{aligned}
E S_{\Delta_{i}}(x)=1 /\left(2 \operatorname{sh} p_{i}\right)\left\{u_{i}\right. & {\left[\exp \left(\sqrt{p_{i} / \varepsilon}\left(h+x-x_{i}\right)\right)-\exp \left(-\sqrt{p_{i} / \varepsilon}\left(x-x_{i-1}\right)\right)\right] } \\
+u_{i-1} & {\left.\left[-\exp \left(\sqrt{p_{i} / \varepsilon}\left(x-x_{i}\right)\right)+\exp \left(\sqrt{p_{i} / \varepsilon}\left(h-\left(x-x_{i-1}\right)\right)\right)\right]\right\} . }
\end{aligned}
$$

Thus,

$$
L S_{\triangle_{i}}(x)=\bar{f}_{i} / \bar{p}_{i}\left\{1+1 /\left(2 \operatorname{sh} \rho_{i}\right)\left(1-\exp p_{j}\right)\left[\exp \left(\sqrt{p_{i} / \varepsilon}\left(x-x_{j}\right)\right)+\exp \left(-\sqrt{p_{i} / \varepsilon}\left(x-x_{i-1}\right)\right)\right]\right\}
$$

and

$$
\left|L\left(E S_{\triangle_{i}}(x)\right)\right| \leq M h^{2}:\left|L\left(L S_{\triangle_{i}}(x)\right)\right| \leq M h^{2}-\bar{f}_{i} / \bar{p}_{i} .
$$

This yields $\left|L\left(u(x)-S_{\triangle_{i}}(x)\right)\right| \leq f / p-\bar{f}_{j} / \bar{p}_{i}+O\left(h^{2}\right)$. Since $\left|\left(\left|p-\bar{p}_{j}\right|,\left|f-\bar{f}_{j}\right|\right)\right| \leq M h^{2}$ we obtain 
$\left|L\left(u(x)-S_{\triangle_{i}}(x)\right)\right| \leq M h^{2}$.

The barrier function $z(x)=C\left(x-x_{i}-h\right)-C h \exp (-\gamma x / \varepsilon) \pm\left(u(x)-S_{\triangle_{i}}(x)\right)$ from [4] gives $L z(x)=C h\left(\gamma^{2} / \varepsilon-p\right) \exp (-\gamma x / \varepsilon)+C p\left(x-x_{i}-h\right) \pm L\left(u(x)-S_{\triangle_{j}}(x)\right)$,

where $p \geq \widetilde{p}>0, C$ and $\gamma$ are constants. Since Theorem 2 then $z\left(x_{i-1}\right) \leq 0, z\left(x_{i}\right) \leq 0$ holds. We can choose $C, \gamma$ so that $L z(x)>0$ because of (10). Then $z(x) \leq 0$ on $\left[x_{i-1}, x_{i}\right]$ from the maximum principle and

$$
\left|u(x)-S_{\triangle_{i}}(x)\right| \leq M h^{2} \text { on }\left[x_{i-1}, x_{i}\right], i<N / 2 .
$$

For $i>N / 2$ we use the second barrier function

$$
z(x)=C\left(x_{i}-x-2 h\right)-C h \exp (-\gamma(1-x) / \varepsilon) \pm\left(u(x)-S_{\triangle_{i}}(x)\right),
$$

and we obtain also the estimate (11)

Remark: The spline solution $S$ of (1) is then

$$
S(x)=\sum_{i=1}^{n} S_{\Delta_{i}}(x) H\left(x_{i}-x\right) \text {. }
$$

where $H$ is the Heaviside function, $H(x)=0$ for $x<0$ and $H(x)=1$ for $x \geq 0$.

Similarly we have the following

Theorem 5: Let $S$ be the spline solution of (1) obtained by (12), where the coefficients $S_{\triangle_{i}}$ in (6) we get from the linear system (7). Let $p, f \in C^{2}[0,1]$. Then the estimate

$$
|u(x)-S(x)| \leq M h^{2} \text { on }[0,1]
$$

holds, where $u$ is the exact solution of (1). It means that the schemes (7) have order two of uniform global convergence.

\section{Numerical results}

In this section we present some numerical examples in order to demonstrate the performance of the schemes (5), (7) for various choices of $\varepsilon$. The calculations were carried out using computer programs written in FORTRAN IV plus in double precision mode, and implemented on (PDP11/34) computer. The numerical rate of the uniform convergence is the following according to Doolan et al [1]:

$$
\text { rate } \equiv\left|\ln z_{i, \varepsilon, h, k}-\ln z_{i, \varepsilon, h, k+1}\right| / \ln 2 ;
$$

where

$$
z_{i, \varepsilon, h, k}=\max _{i}\left|u_{i, \varepsilon, h / 2^{k}}-u_{i, \varepsilon, h / 2^{k+1}}\right|, k=0(1) 6 .
$$

Here $u_{i, \varepsilon, h / 2^{k}}$ is the approximate value at the point $x_{i}$, length $h / 2^{k}$. The meshes were obtained by halving previous ones successively starting with 16 points and ending with 1024 . We tested the simple example

$$
-\varepsilon u^{\prime \prime}(x)+u(x)=\cos ^{2} \pi x+2 \varepsilon \pi^{2} \cos 2 \pi x, u(0)=u(1)=0
$$

with the exact solution 


$$
u(x)=(\exp (-(1-x) / \sqrt{\varepsilon})+\exp (-x / \sqrt{\varepsilon})) /(1+\exp (-1 / \sqrt{\varepsilon}))-\cos ^{2} \pi x
$$

on the schemes (5), (7). The resulting values and nodal errors are displayed in the following tables (in which $\mathrm{N}$ stands for $\mathrm{N}$ ). The maximum difference between two consecutive meshes is listed in the $E_{\infty}$ norm $\left(=z_{i, c, h, k}\right)$. The declination of the approximate solution from the exact one is listed in the maximum norm and denoted by $\gamma_{i, \varepsilon, h}=\max _{i}\left|u_{i, \varepsilon, h}-u_{\varepsilon}\left(x_{i}\right)\right|$.

Table 1: Rates of convergence for (7), and max differences

\begin{tabular}{|c|c|c|c|c|c|c|c|c|c|c|}
\hline $\mathbb{N}$ & 16 & & 32 & & 64 & & 128 & & 256 & 512 \\
\hline \multirow[t]{2}{*}{$\varepsilon \backslash K$} & & 1 & & 2 & & 3 & & 4 & & 5 \\
\hline & $E_{\infty}$ & rate & $E_{\infty}$ & rate & $E_{\infty}$ & rate & $E_{\infty}$ & rate & $E_{\infty}$ & rate \\
\hline \multirow[t]{2}{*}{1} & $.11 E-02$ & & $29 E-03$ & & $.71 E-04$ & & $.18 E-04$ & & $44 E-05$ & \\
\hline & & 2.02 & & 2.01 & & 2.00 & & 2.00 & & 2.00 \\
\hline \multirow[t]{2}{*}{$2^{-1}$} & $.19 E-02$ & & $.27 E-03$ & & $.68 E-04$ & & $17 E-04$ & & $42 E-05$ & \\
\hline & & 2.02 & & 2.01 & & 2.00 & & 2.00 & & 2.00 \\
\hline \multirow[t]{2}{*}{$2^{-2}$} & $.99 E-03$ & & $25 E-03$ & & $62 E-04$ & & $16 E-04$ & & $.39 E-05$ & \\
\hline & & 2.02 & & 2.01 & & 2.00 & & 2.00 & & 2.00 \\
\hline \multirow[t]{2}{*}{$2^{-3}$} & $88 E-03$ & & $22 E-03$ & & $55 E-04$ & & $14 E-04$ & & $.34 E-05$ & \\
\hline & & 2. 02 & & 2.01 & & 2.00 & & 2.00 & & 2.00 \\
\hline \multirow[t]{2}{*}{$2^{-4}$} & $.77 E-03$ & $\cdot$ & $.19 E-03$ & & $.48 E-04$ & & $12 E-04$ & & $.30 E-05$ & \\
\hline & & 2.03 & & 2.01 & & 2.00 & & 2.00 & & 2.00 \\
\hline \multirow[t]{2}{*}{$2^{-5}$} & $.68 E-03$ & & $.17 E-03$ & & $42 E-04$ & & $.11 E-04$ & & $.26 E-05$ & \\
\hline & & 2.04 & & 2.01 & & 2.00 & & 2.00 & & 2.00 \\
\hline \multirow[t]{2}{*}{$2^{-6}$} & $.63 E-03$ & & $.16 E-03$ & & $39 E-04$ & & $98 E-05$ & & $.24 E-05$ & \\
\hline & & 2.05 & & 2.01 & & 2.00 & & 2.00 & & 2.00 \\
\hline \multirow[t]{2}{*}{$2^{-7}$} & $62 E-03$ & & $15 E-03$ & & $.38 E-04$ & & $95 E-05$ & & $.24 E-05$ & \\
\hline & & 2.08 & & 2.02 & & 2.01 & & 2.00 & & 2.00 \\
\hline \multirow[t]{2}{*}{$2^{-8}$} & $62 E-03$ & & $15 E-03$ & & $.38 E-04$ & & $94 E-05$ & & $24 E-05$ & \\
\hline & & 2.14 & & 2.04 & & 2.01 & & 2.00 & & 2.00 \\
\hline \multirow[t]{2}{*}{$2^{-9}$} & $.64 E-03$ & & $15 E-03$ & & $.38 E-04$ & & $.94 E-05$ & & $.24 E-05$ & \\
\hline & & 2.23 & & 2.07 & & 2.02 & & 2.00 & & 2.00 \\
\hline \multirow[t]{2}{*}{$2^{-10}$} & $.68 E-03$ & & $.16 E-03$ & & $.38 E-04$ & & $94 E-05$ & & $24 E-05$ & \\
\hline & & 2.36 & & 2.12 & & 2.04 & & 2.01 & & 2.00 \\
\hline \multirow[t]{2}{*}{$2^{-21}$} & $74 E-03$ & & $.16 E-03$ & & $.38 E-04$ & & $95 E-05$ & & $24 E-05$ & \\
\hline & & 2.51 & & 2.22 & & 2.07 & & 2.02 & & 2.00 \\
\hline \multirow[t]{2}{*}{$10^{-4}$} & $.11 E-02$ & & $.19 E-03$ & & $.41 E-04$ & & $.96 E-05$ & & $.24 E-05$ & \\
\hline & & 2.53 & & 2.54 & & .225 & & 2.08 & & 2.02 \\
\hline \multirow[t]{2}{*}{$10^{-5}$} & $.18 E-02$ & & $.37 E-03$ & & $.60 E-04$ & & $.11 E-04$ & & $.25 E-05$ & \\
\hline & & 2.02 & & 2.28 & & 2.60 & & 2.44 & & 2.17 \\
\hline
\end{tabular}


Table 2: Rates and max differences for (7)

\begin{tabular}{|c|c|c|c|c|c|c|c|c|c|c|}
\hline $\mathbb{N}$ & 16 & & 32 & & 64 & & 128 & & 256 & 512 \\
\hline$\varepsilon \backslash K$ & & 1 & & 2 & & 3 & & 4 & & 5 \\
\hline & $E_{\infty}$ & rate & $E_{\infty}$ & rate & $E_{\infty}$ & rate & $E_{\infty}$ & rate & $E_{\infty}$ & rate $E_{\alpha}$ \\
\hline 1 & $.11 E-02$ & 2.01 & $.28 E-03$ & 2.00 & $.71 E-04$ & 2.00 & $.18 E-04$ & 2.00 & $.44 E-0$ & 2.00 \\
\hline $10^{-2}$ & $.60 E-03$ & 1.95 & $15 E-03$ & 1.99 & $.38 E-04$ & 2.00 & $.95 E-05$ & 2.00 & $.24 E-0$ & 2.00 \\
\hline $10^{-5}$ & $.57 E-03$ & 2.08 & $.64 E-04$ & 3.15 & $15 E-04$ & 2.10 & $.77 E-05$ & 2. 49 & $.22 E-C$ & .179 \\
\hline
\end{tabular}

Table 3: Errors $\gamma_{i, c, h}$ for (7),

\begin{tabular}{|c|cccccc|}
\hline$E \backslash N$ & 16 & 32 & 64 & 128 & 256 & 512 \\
\hline $2^{-9}$ & $.85 E-03$ & $.20 E-03$ & $.50 E-04$ & $.13 E-04$ & $.31 E-05$ & $.78 E-06$ \\
\hline
\end{tabular}

Table 4: Rates and errors in $E_{c o}$ norm for (5),

\begin{tabular}{|c|c|c|c|c|c|c|c|c|c|c|}
\hline $\mathbb{N}$ & 16 & & 32 & & 64 & & 128 & & 256 & \\
\hline$E \backslash K$ & & 1 & & 2 & & 3 & & 4 & & 5 \\
\hline \multirow[t]{2}{*}{1} & $.11 E-02$ & & $29 E-04$ & & $71 E-04$ & & $.18 E-04$ & & $.44 E-05$ & \\
\hline & & 2.02 & & 2.01 & & 2.00 & & 2.00 & & 2.00 \\
\hline \multirow[t]{2}{*}{$10^{-2}$} & $.62 E-03$ & & $15 E-03$ & & $38 E-04$ & & $.95 E-05$ & & $.24 E-05$ & \\
\hline & & 2.07 & & 2.02 & & 2.01 & & 2.00 & & 2.00 \\
\hline \multirow[t]{2}{*}{$10^{-4}$} & $.11 E-02$ & & $.19 E-03$ & & $.41 E-04$ & & $.96 E-05$ & & $.24 E-05$ & \\
\hline & & 2.53 & & 2.54 & & 2.25 & & 2.08 & & 2.02 \\
\hline \multirow[t]{2}{*}{$10^{-5}$} & $.18 E-02$ & & $.37 E-03$ & & $.60 E-04$ & & $11 E-04$ & & $.25 E-05$ & \\
\hline & & 2.02 & & 2.28 & & 2.60 & & 2.44 & & 2. 17 \\
\hline
\end{tabular}


Table 5: Rates and errors in $E_{\infty}$ norm for $(5)_{2}$

\begin{tabular}{|c|c|c|c|c|c|c|c|c|c|c|}
\hline oN & 16 & & 32 & & 64 & & 128 & & 256 & \\
\hline$\varepsilon \backslash \mathrm{K}$ & & 1 & & 2 & & 3 & & 4 & & 5 \\
\hline \multirow[t]{2}{*}{1} & $.11 E-02$ & & $.28 E-03$ & & $.71 E-04$ & & $18 E-04$ & & $44 E-05$ & \\
\hline & & 2.01 & & 2.00 & & 2.00 & & 2.00 & & 2.00 \\
\hline \multirow[t]{2}{*}{$10^{-3}$} & $.53 E-03$ & & $.15 E-03$ & & $.37 E-04$ & & $94 E-05$ & & $24 E-05$ & \\
\hline & & 1.04 & & 1.87 & & 1.97 & & 1.99 & & 2.00 \\
\hline \multirow[t]{2}{*}{$10^{-5}$} & $.57 E-03$ & & $.64 E-04$ & & $15 E-04$ & & . $18 E-05$ & & $22 E-05$ & \\
\hline & & 2.08 & & 3.15 & & 2.10 & & 95 & & 1.79 \\
\hline
\end{tabular}

Table 6: Errors $\gamma_{i, \varepsilon, l}$ for $(5)_{1}$

\begin{tabular}{|c|cccccc|}
\hline$E \backslash^{\mathbb{N}}$ & 16 & 32 & 64 & 128 & 256 & 512 \\
\hline $10^{-5}$ & $.22 E-03$ & $.41 E-03$ & $.75 E-04$ & $.14 E-04$ & $.33 E-05$ & $.79 E-06$ \\
\hline
\end{tabular}

\section{REFERENCES}

[1] DoolAN. E.P., MILLER, J.J.H., and W.H. A. SCHILdERS: Uniform Numerical Methods for Problems with Initial and Boundary Layers. Dublin: Boole Press 1980

[2] FARRELL, P. A.: Uniform and optimal schemes for stiff initial-value problems. Comput. Math. Appl. 13 (1987), $925-936$.

[3] HEMKER, P.W.: Nemerical aspects of singular perturbation problems. Lect. Notes Math. 985 (1983). $267-287$

[4] ORIORDAN, E., and M. STYNES: 1 uniformly accurate finite element method for a singularly perturbed one-dimensional reaction diffusion problem. Math. Comput. 47 (1986). $555-570$.

[5] SURLA, K., and M. STOJANOVIC: A solving singularly perturbed boundary value problems by spline in tension. J. Comput. Appl. Math. 24 (1988), 355 - 363.

[6] STOJANOVIĆ. M.: Spline collocation method. Mat. Glas. (to appear).

Received 16.08.1990; in revised form 23.07.1991

Dr. Mirjana Stojanovic:

lustitute of Mathematics of the University

Trg Dositeja Obradovica 4

YU - 21000 Novi Sad 